\title{
LES GARANTIES JURIDIQUES DE L'ALTERNANCE POLITIQUE EN REPUBLIQUE DEMOCRATIQUE DU CONGO
}

\author{
Par Joseph Cihunda Hengelela ${ }^{1}$
}

Tshilema, mweene wa dinyingika ${ }^{2}$.

Proverbe Tshokwe

\section{Résumé}

Cet article analyse les garanties juridiques de l'alternance politique démocratique en RDC sous la Constitution du 18 février 2006. Il démontre que suivant l'ordre constitutionnel en vigueur, rien ne peut empêcher l'alternance politique en RDC. Ainsi tout projet visant à faire obstacle à l'alternance politique constitue une infraction imprescriptible de renversement du régime constitutionnel établi. Il est en lui-même une menace sérieuse à la paix et à la sécurité tant de la RDC que de toute l'Afrique des Grands Lacs.

\section{INTRODUCTION}

L'alternance en politique est entendue comme le processus par lequel un parti politique ou un groupe des partis politiques appartenant à un courant idéologique différent succède à un autre au pouvoir à l'issue des élections libres et transparentes. Ainsi l'alternance politique est considérée comme une condition nécessaire, mais sans être suffisante, de la démocratie. D'où le concept « alternance démocratique ». Ce concept suggère l'idée selon laquelle qu'il peut y avoir une alternance sans que celle-ci soit démocratique. Pour saisir la portée de l'alternance démocratique, il importe de rechercher sa définition telle qu'elle apparait dans le vocabulaire politique.

Suivant le dictionnaire Toupie, l'alternance est l'« action d'alterner, de succéder dans le temps de manière régulière ». Associée à la politique, l'alternance « correspond à une situation d'un régime politique où des courants, des tendances ou des partis politiques différents se succèdent au pouvoir. C'est le cas lorsque la majorité politique est renversée par l'oppo-

1 Diplômé d'Etudes Supérieures en Droit Public et Doctorant/Université de Kinshasa et membre du CODESRIA.

Consultant et Avocat au Barreau de Kinshasa/Matete, Email : josephcihunda@gmail.com; jcihunda@yahoo.fr

Tél. : 00243810399060 / 976008034.

2 Littéralement : un infirme, se connaît lui-même. En d'autres termes, lorsqu'on connaît ses propres faiblesses, on sait comment les prévenir ainsi que les conséquences qu'elles peuvent engendrer. 
sition, dans le respect des règles constitutionnelles, lors d'une élection législative ou présidentielle $» .^{3}$

Cette définition ne fait pas la distinction entre l'alternance des partis politiques et celle des individus appartenant à un même parti politique. En effet, une alternance démocratique peut avoir lieu sans que le parti politique au pouvoir ne perde les élections. Il s'agit d'une alternance des personnes qui n'est pas sans avoir avec les programmes politiques du parti au pouvoir. Deux exemples peuvent être cités pour souligner quelques limites que l'on peut reprocher à la définition donnée de l'alternance politique.

Le premier exemple est celui de la Tanzanie où le parti politique Chama cha Mapinduzi (CCM) remporte toutes les élections depuis le retour du multipartisme dans ce pays. Si le programme politique est resté pratiquement le même (socialisme africain-Ujama) en dépit de ses inflexions vers la social-démocratie, il y a eu une alternance des personnes à la tête de ce pays d'Afrique des Grands Lacs. Le deuxième exemple est celui tiré de l'expérience de l'Afrique du Sud postapartheid. Dans ce pays, l'African National Congress (ANC) remporte toutes les élections depuis 1994. On peut affirmer l'existence de l'alternance des personnes à la tête de cet Etat. Beaucoup d'interrogations restent posées au sujet du programme politique de ce parti de Nelson Mandela. Une simple observation renseigne que la politique menée par Nelson Mandela et Thabo Mbeki ${ }^{4}$ est différente de celle de Jacob Zuma, plus particulièrement en ce qui concerne la politique africaine de l'Afrique du Sud et la politique de l'immigration. ${ }^{5}$

Tout compte fait, l'alternance politique ne signifie pas seulement le changement de position entre les partis politiques allant de l'opposition au pouvoir et vice versa, mais aussi de changement du contenu des programmes politiques ou de gouvernement et des personnes chargées de les mettre en œuvre. C'est cette dimension qui nous préoccupe dans le cadre de cette réflexion. De cette définition de l'alternance, il s'impose l'établissement des rapports d'abord, entre l'alternance politique et la démocratie, ensuite entre l'alternance politique et l'Etat de droit et enfin, entre l'alternance politique et la stabilité politique, économique et sécuritaire.

Au sujet des rapports entre l'alternance politique et la démocratie, le dictionnaire Toupie soutient que

la possibilité qu'il y ait une alternance politique est l'une des conditions nécessaires à la démocratie. Elle témoigne en effet de l'existence de libertés politiques et d'un

3 «Alternance », http://www.toupie.org/Dictionnnaire/Alternance (Consulté le 19 septembre 2014).

4 Voir Mboya Mzobanzi M., Mbeki and the Peace Process in Africa : A Contribution to Africa's Renaissance, International Journal of African Renaissance Studies, Volume 1, N 1, 2006, pp. 80-89; Kabemba C., South Africa in the DRC : renaissance or néo-imperialism?, in Sakhela Buhlungu et al (ed.), State of the Nation. South Africa 2007, Cape Town, 2007, p. 536 (pp. 533-551). Voir aussi Manby B., La nationalité en Afrique, Paris, 2011, p. 205.

5 Abahlali baseMjondolo, SA xenophobic attacks: A view from below, http://pambazuka.org/en/categ ory/features/94476 (Consulté le 15 avril 2015). 
régime pluraliste où le parti au pouvoir accepte de se retirer en cas de défaite électorale. L'alternance a pour conséquence de renforcer la légitimité de la Constitution et l'adhésion des citoyens au régime politique ${ }^{6}$.

Ainsi donc, l'alternance politique est une des composantes majeures de la démocratie. Il n'y a pas de démocratie sans alternance au pouvoir. Mais elle ne suffit pas à elle seule à établir une démocratie véritable. Il faut que l'alternance politique amène à l'amélioration des conditions de vie des citoyens et au développement du pays en général. Comme le souligne Delly Sesenga, une vraie alternance politique doit aboutir à une alternative politique au pouvoir. $^{7}$

Jean-Louis Quermone abonde dans le même sens lorsqu'il affirme que « L'alternance offre aux citoyens l'occasion d'évaluer les performances des politiques menées par leurs dirigeants. Et elle tend ainsi à assurer l'exigence de 'gouvernement pour le peuple' à l'exercice du 'gouvernement par le peuple'. ${ }^{8}$ L'alternance politique s'effectue dans le strict respect du régime constitutionnel en vigueur. Elle permet d'opérer « un changement de rôle entre les forces politiques situées dans l'opposition qu'une élection au suffrage universel fait accéder au pouvoir, et d'autres forces politiques qui y renoncent provisoirement pour entrer dans l'opposition ». L'alternance politique participe à la consolidation de la démocratie. ${ }^{9}$

Le rapport existant entre l'alternance politique et l'Etat de droit peut être établi par le fait que lorsque l'on évoque l'idée d'alternance en politique, il est supposé le dépassement $\mathrm{du}$ stade où règne le primat de l'individu détenteur du pouvoir sur les textes juridiques. Au contraire, l'Etat de droit suppose le primat de la loi sur les individus. Ce dernier est celui où l'alternance politique au pouvoir se déroule conformément aux prescrits de la Constitution ou de la loi fondamentale; car ce qui importe n'est pas la personne qui détient le pouvoir ou celle qui va lui succéder mais le respect absolu des textes juridiques, plus particulièrement de la Constitution.

Selon Tshikala Biaya, dans tout Etat démocratique, « ...l'Etat de droit repose sur trois piliers : la légitimité des gouvernants, la séparation des pouvoirs et l'indépendance de la magistrature... ». ${ }^{10}$ La légitimité des gouvernants est obtenue par l'accession au pouvoir conformément aux textes juridiques en vigueur et à la gestion des affaires publiques selon

6 Alternance, http://www.toupie.org/Dictionnnaire/Alternance (Consulté le 19 septembre 2014).

7 Sesanga Hipungu D., La voie du changement. Un pari de la raison pour la Rd Congo, Paris, 2011, p. 51.

8 Quermone J.L., L'alternance au pouvoir, Paris, 2003, p. 8 cité par Kamukuny Mukinay A., Contribution à l'étude de la fraude en droit constitutionnel congolais, Thèse de doctorat, Faculté de droit, Université de Kinshasa, 2007, p. 466.

9 Quermone J.L., L'alternance au pouvoir....op. cit. Voir aussi Etekou Bédi Y.S., L'alternance démocratique dans les Etats d'Afrique francophone, Thèse de doctorat, Université de Paris-Est et Université de Cocody, 2013.

10 Biaya T.K., Quelle Démocratie pour l'Afrique? Réflexions sur ses Possibilités et ses Caractéristiques, Dakar, 1998, voir http://unpan1.un.org (Consulté le 25 avril 2015). 
la volonté du souverain primaire. ${ }^{11}$ En effet, la légitimité des gouvernants est renforcée par l'alternance au pouvoir. Ainsi l'on ne peut pas prétendre être dans un Etat de droit et faire obstacle à l'alternance au pouvoir. Le constituant du 18 février 2006 en RDC était bien conscient de ce rapport qu'il y a entre l'Etat de droit et l'alternance politique au pouvoir. Il a non seulement déclaré que la RDC est un Etat de droit mais surtout avoir prévu des mécanismes pour assurer cette alternance politique. ${ }^{12}$

A propos des liens entre l'alternance politique, la stabilité politique, la paix et le développement, il convient de soutenir que l'alternance au pouvoir est devenue aujourd'hui un gage et une condition de la stabilité politique, économique et sécuritaire dans la plupart des pays africains. Le fait de rester longtemps au pouvoir devient une cause de l'instabilité politique, de stagnation économique et une source des conflits politiques et armés. ${ }^{13}$ Dans ce contexte, l'alternance politique au pouvoir est et demeure une garantie à la stabilité des institutions politiques, de la croissance économique et de la paix. Il est supposé que la lutte pour accéder au pouvoir est « civilisée » et l'accès aux institutions étatiques est garanti pour tout citoyen qui remplit les conditions fixées par la Constitution et les lois de la République. Le renouvellement régulier des animateurs est susceptible d'accélérer le développement du pays.

Cette réflexion est limitée à l'alternance au niveau de l'institution Président de la République compte tenu de l'influence que celle-ci a sur les autres institutions politiques dans un Etat. Elle est construite autour des questions de savoir si la Constitution du 18 février 2006 est-elle une réponse aux crises de politiques de la RDC? A-t-elle prévu des mécanismes pour l'alternance politique, étant entendu que cette dernière est la cause des crises de légitimité qui ont caractérisé la vie des institutions politiques durant les cinquante dernières années? Au bout du compte, quelles sont les atteintes et les tentatives d'atteintes à l'alternance politique depuis l'entrée en vigueur de la Constitution du 18 février 2006 en RDC?

11 Je voudrais souligner le fait que la légitimité n'est pas une donnée constante. Elle est variable. Elle peut augmenter comme dégringoler. Lorsqu'elle dégringole, l'on se trouve dans l'hypothèse de l'érosion ou de l'effritement de la légitimité. Tout dépend du comportement du détenteur du pouvoir.

12 Vunduawe te Pemako F. et Mboko Dj'andima J.M., Droit constitutionnel du Congo. Textes et documents fondamentaux, Vol. 2, Bruxelles, 2013, p. 1021.

13 Les tentatives de faire obstacles à l'alternance politique sont à l'origine des violences en ce début de l'année 2015 au Burundi et en RDC. Voir http://radiookapi.net/actualite/2015/01/19/rdc-plusieu rs-activites-perturbees-kinshasa-dans-les-manifestations-contre-la-loi-electorale/, (Consulté le 25 avril 2015); Kibangula T., Burundi : au $3^{\text {ème }}$ jour de manifestations, le pouvoir de Nkurunziza parle d"'insurrection", http://www.jeuneafrique.com/Article/ARTJAWEB20150428152155/, (Consulté le 30 avril 2015). 


\section{A. La Constitution du 18 février 2006 en tant qu'une réponse aux crises politiques de la $R D C$}

La RDC est un pays en crise perpétuelle depuis sa naissance en tant qu'Etat indépendant et souverain. En effet, après la proclamation de l'indépendance le 30 juin 1960, la Force publique s'était mutinée le 5 juillet de la même année, soit cinq jours après l'indépendance. La mutinerie a été suivie par les sécessions des provinces du Katanga et du Sud-Kasaï et la révocation du Premier ministre Emery Patrice Lumumba par le Chef de l'Etat Joseph Kasavubu sur fond de l'interprétation erronée de l'article 22 de la Loi fondamentale ${ }^{14}$. C'est là le début de la crise de légitimité qui a duré pendant quarante-six ans et qui n'a pas permis aux différents gouvernements successifs de bâtir le développement économique, social et culturel du pays.

La Constitution du 18 février 2006, rédigée conformément aux recommandations du Dialogue inter congolais, était conçue comme une réponse adéquate à la crise de légitimité des dirigeants et une prise en charge des causes profondes des crises politiques en RDC. Pour le constituant originaire, la cause de crises politiques récurrentes est la « contestation de la légitimité des Institutions et de leurs animateurs. Cette contestation a pris un relief particulier avec les guerres qui ont déchiré le pays de 1996 à $2003 »{ }^{15}$ En réalité, la contestation de la légitimité par le recours aux moyens militaires date des années 1960 à travers des rébellions telles que celles menées par le Conseil National de Libération (CNL) et des guerres contre le régime du maréchal Mobutu comme la guerre de quatre-vingts jours ou celles dites de Shaba. ${ }^{16}$

Ce qui importe plus est le but poursuivi par le constituant congolais tourmenté par les cendres fumantes de la tragédie que d'aucuns n'ont pas hésité de qualifier de " première guerre mondiale africaine ». En effet, le constituant du 18 février 2006 s'était fixé comme objectifs entre autres de « ...mettre fin à cette crise chronique de légitimité et de donner au pays toutes les chances de se reconstruire ». Comment cet objectif allait-il être matérialisé? Le constituant avait réservé une réponse à cette question. Pour lui, la seule solution est la mise en place d'un

14 Art. 22 de la Loi fondamentale du 19 mai 1960 relative aux structures du Congo stipulait : " Le Chef de l'État nomme et révoque le Premier Ministre et les Ministres ». Dans ce régime parlementaire qu'elle avait établi, le Chef de l'Etat ne pouvait nommer ou révoquer que sur proposition de la Chambre des Représentants. Ce qui n'a pas été le cas. Le Chef de l'Etat Joseph Kasavubu révoqua unilatéralement le Premier ministre Patrice Emery Lumumba malgré la pleine confiance que ne cessait de lui témoigner l'ensemble des parlementaires. Celui-ci révoqua à son tour le Chef de l'Etat sans soubassement juridique. C'est le début d'une crise politique qui dura longtemps.

$15 \S 1$ de l'exposé des motifs de la Constitution de la République Démocratique du Congo, JORDC,

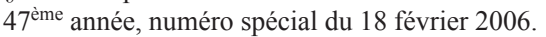

16 La guerre dite de Shaba I et Shaba II sont des incursions militaires menées par les gendarmes katangais réfugiés en Angola depuis la fin de la sécession de la province du Katanga en 1963. Voir Mobutu, Dignité pour l'Afrique. Entretiens avec Jean-Louis Remilleux, Paris, 1989, pp. 73-75. Ce contexte de guerre de Shaba avait inspiré roman à un figure de proue de la littérature congolaise : Valentin Yves Mudimbe, Shaba deux. Les Carnets de Mère Marie Gertrude, Paris, 1989. 
nouvel ordre politique, fondé sur une nouvelle Constitution démocratique sur base de laquelle le peuple congolais puisse choisir souverainement ses dirigeants, au terme des élections libres, pluralistes, démocratiques, transparentes et crédibles. ${ }^{17}$

Cependant, le constituant n'avait pas défini ce qu'il entendait par le « nouvel ordre politique » (NOP). Il s'est milité seulement à indiquer que cet ordre politique est fondé sur une nouvelle Constitution démocratique. L'un des éléments le plus ostensible de cet ordre politique est le choix par le peuple congolais de ses dirigeants à travers des élections libres, pluralistes, démocratiques, transparentes et crédibles. Il devient impérieux de rechercher les éléments constitutifs du nouvel ordre politique congolais dans la Constitution qui en constitue le fondement juridique.

Il parait que le constituant du 18 février 2006 avait regroupé les éléments constitutifs du nouvel ordre politique de la RDC autour de cinq points majeurs. Il s'agit de la souveraineté du peuple, de la structure administrative de l'Etat, des droits, des libertés et des devoirs des citoyens, de l'organisation et de l'exercice du pouvoir et de la révision constitutionnelle. De la souveraineté du peuple congolais, dérivent les principes du nouvel ordre politique suivants : l'émanation de tout pouvoir du peuple en tant que souverain primaire, le choix souverain des dirigeants par des élections régulières, le pluralisme politique et l'unicité et l'exclusivité de la nationalité congolaise. ${ }^{18}$

Au sujet de la structure administrative, les éléments du nouvel ordre politique sont l'organisation territoriale de l'Etat en 25 provinces plus la ville de Kinshasa dotées toutes de la personnalité juridique et exerçant des compétences politiques de proximité; la retenue à la source dans chaque province de $40 \%$ de recettes à caractère national et la disposition par les provinces des organes politiques (Gouvernements provinciaux et Assemblées provinciales). ${ }^{19}$

En ce qui concerne les droits humains, les libertés fondamentales et les devoirs des citoyens, le constituant du 18 février 2006 avait exprimé son attachement aux droits humains et aux libertés fondamentales proclamés dans les instruments juridiques internationaux auxquels la RDC a adhéré. Ces droits et libertés ont été intégrés dans la Constitution jusqu'à la formalisation de la parité homme-femme. Dans le titre consacré aux droits humains, le constituant du 18 février 2006 a innové en consacrant l'intangibilité de certains droits humains dont le droit à la vie et enrichi la nomenclature des droits sociaux protégés par la Constitution. ${ }^{20}$ A côté de ces droits, le constituant a imposé des devoirs aux citoyens congolais pour préserver ce nouvel ordre politique. L'un de devoirs le plus caractéristique du nou-

$17 \S 2$ de l'exposé des motifs de la Constitution de la République Démocratique du Congo,...op. cit.

18 Art. 5 de la Constitution de la République Démocratique du Congo, Journal Officiel de RDC (JORDC), $47^{\text {ème }}$ année, numéro spécial du 18 février 2006.

19 Art. 2, 3, 4, 175, 195-206 de la de la Constitution de la République Démocratique du Congo...op. cit.

20 Art. 11 à 61 de la Constitution de la République Démocratique du Congo...op. cit. 
vel ordre politique congolais est celui de faire échec à la prise du pouvoir par la force ou à son exercice en violation de la Constitution. ${ }^{21}$

De l'organisation et de l'exercice du pouvoir, il découle les éléments suivants : « assurer le fonctionnement harmonieux des Institutions de l'Etat, éviter les conflits, instaurer un Etat de droit, contrer toute tentative de dérive dictatoriale, garantir la bonne gouvernance, lutter contre l'impunité et assurer l'alternance démocratique. ${ }^{22}$ Le cinquième point du nouvel ordre politique congolais est relatif à la révision constitutionnelle qui ne peut désormais s'appliquer à une catégorie des matières. Il découle de ce point un seul élément : l'intangibilité des dispositions relatives à la forme républicaine de l'Etat, au principe du suffrage universel, à la forme représentative du Gouvernement, au nombre et à la durée des mandats du Président de la République, à l'indépendance du pouvoir judiciaire, au pluralisme politique et syndical. ${ }^{23}$ L'alternance politique est un élément constitutif du nouvel ordre politique et constitutionnel congolais.

\section{B. Les mécanismes garantissant l'alternance politique dans la Constitution du 18 février 2006}

Les mécanismes constitutionnels tendant à promouvoir et à garantir l'alternance politique en RDC peuvent être rangés selon qu'ils se rapportent au mandat présidentiel, au statut des anciens Présidents de la République et au devoir de résistance à la prise du pouvoir par la force ou à son exercice en violation de la Constitution. Ces garanties ne concernent que l'alternance à la fonction de Président de la République, choisie comme objet de cette réflexion et compte tenu du débat que cette question a donné lieu dans l'opinion publique tant nationale, africaine qu'internationale.

\section{La limitation du nombre et de la durée des mandats présidentiels}

La limitation du nombre et la durée des mandats présidentiels est une des réponses, sinon la principale, à la crise de légitimité en RDC. En évitant qu'un homme se maintienne au pouvoir le plus longtemps possible, le constituant du 18 février 2006 voulait épargner l'Etat de l'usure du pouvoir qui constitue un frein au développement politique, économique et social. ${ }^{24}$ Deux dispositions constitutionnelles prennent en charge cette matière de limitation du nombre des mandats présidentiels et leur durée. Il s'agit des articles 70 et 220.

21 Art. 62 à 67 de la Constitution de la République Démocratique du Congo...op. cit.

22 Voir exposé des motifs de la Constitution de la République Démocratique du Congo...op. cit.

23 Art. 220 de la Constitution de la République Démocratique du Congo...op. cit.

24 Kaniki F., Discours de Barack OBAMA : Une arme pour l'alternance politique en Afrique, cite un extrait du discours d'Obama: “...un dirigeant qui s'accroche longtemps au pouvoir,à la fin le dirigeant agit pour durer et non pour ce qui concerne le pays. Et ne vous laissez pas duper par la notion selon laquelle il y aurait une voie africaine. Faire les choses à l'africaine ce n'est pas arriver au pouvoir et d ' un coup avoir deux milliards de dollars sur un compte en banque en Suisse". http:/ /afrique.kongotimes.info/afrique (Consulté le 29 avril 2015). 
L'article 70 dispose :

Le Président de la République est élu au suffrage universel direct pour un mandat de cinq ans renouvelable une seule fois.

A la fin de son mandat, le Président de la République reste en fonction jusqu'à l'installation effective du nouveau Président élu.

Cette disposition constitutionnelle mérite des commentaires tendant à apporter des précisions en vue de contribuer à la dissipation de toute interprétation erronée dans le contexte où le juge compétent, en l'occurrence la Cour constitutionnelle et bien avant elle, la Cour suprême de Justice (CSJ), ne semble pas donner les garanties d'indépendance à l'égard du pouvoir exécutif. ${ }^{25}$

Si la durée du mandat présidentiel (cinq ans) ne pose pas de problème, il n'en est pas le cas pour le nombre dudit mandat. Le constituant du 18 février 2006 a été plus clair et précis. Ce mandat d'une durée de cinq ans est renouvelable une seule fois. L'idée de limiter le mandat présidentiel est à trouver notamment dans le projet de Constitution de la CNS. L'article 105 de cet projet disposait que « Le Président de la République est élu pour cinq ans au suffrage universel direct et secret. Il n'est rééligible qu'une seule fois... $»{ }^{26}$

Contre cette volonté concise et constante du constituant congolais, il se développe dans les discours distillés dans l'opinion publique la thèse de «mandats présidentiels non successifs ». Suivant cette thèse, un ancien Président de la République qui avait épuisé ses deux mandats, peut revenir au pouvoir. Des zones d'ombres demeurent nombreuses autour de cette thèse. Les partisans de cette thèse ne disent pas après combien de temps, un ancien Président de la République peut prétendre au fauteuil présidentiel et quelles conditions qu'il doit remplir pour ce faire?

25 La jurisprudence de la Cour suprême de Justice en matière constitutionnelle démontre l'absence d'indépendance de cette juridiction à l'égard du pouvoir exécutif. Voir Akele Adau P., La question de la qualité militaire du Président Joseph Kabila au regard de la loi sur l'identification et l'enrôlement des électeurs, Congo-Afrique (Octobre 2005) n 398, pp. 431-441; Wetsh'okonda Koso M., Les textes constitutionnels congolais annotés, Kinshasa, 2010; Balingene Kahombo, La Cour suprême de Justice, Cour constitutionnelle transitoire, http://www.la-constitution-en-afrique.org/, (Consulté le 2 février 2011); CSJ, 15 septembre 2006, Arrêt R.Const. 37/TSR, en cause la CEI, Bulletin des arrêts de la CSJ...op. cit., p. 395; CSJ, 27 novembre 2006, Arrêt RCE.PR. 009, en cause Recours du MLC, Bulletin des arrêts de la CSJ...op. cit., pp. 83-110; CSJ, 13 avril 2006, Arrêt RCDC 003/KIN, en cause MSD contre CEI, in Bulletin des arrêts de la CSJ...op. cit., pp. 3-5; CSJ, 16 décembre 2011, R.E.007, cinquième feuillet, inédit. Dans le même sens, Kifwabala Tekilazaya, Fataki wa Luhindi D. et Wetsh'okonda Koso M., République Démocratique du Congo. Le secteur de la justice et l'Etat de droit, Johannesbourg, 2013, p. 16; Mumbere Bamlango D., Des actes législatifs et du pouvoir régulateur : quand la Cour suprême de Justice donne une portée expansive aux attributions de la Cour constitutionnelle, Horizons, Revue de droit et science politique du Graben, $n^{\circ} 5$, pp. 46-58.

26 Art. 105 du Projet de Constitution de la République fédérale du Congo, Palais du Peuple, Kinshasa, 1992. 
Cette thèse ne semble pas tenir debout. Elle semble avoir été échafaudée dans un contexte plus particulier; celui de la recherche des stratagèmes pour opérer une fraude à la Constitution et occasionner une détention du pouvoir d'Etat par une personne qui pourrait le céder à qui elle veut tout en conservant son contrôle et le reprendre plus tard. ${ }^{27}$ Cette thèse est calquée sur le style russe où le pouvoir d'Etat est échangé depuis 2000 entre Vladimir Vladimirovitch Poutine et Dmitri Anatolievitch Medvedev devenant tour à tour Président de la République et Premier ministre de l'un et de l'autre. Il en est de même du modèle argentin en dépit du fait que celui-ci s'est déroulé dans un contexte démocratique. En Argentine, le pouvoir présidentiel est passé de Néstor Carlos Kirchner (2003-2007) à sa femme Cristina Fernandez de Kirchner. Pressenti pour revenir Nestor au pouvoir Carlos Kirchner est mort en 2010 avant la fin du mandat de son épouse.

Contrairement à ces pratiques de succession au pouvoir, le constituant congolais avait prescrit que le mandat présidentiel est renouvelable une et une seule fois pour toute. En d'autres termes, la durée maximale à la fonction présidentielle est de dix ans pour tout Congolais ayant été choisi pour assumer cette charge publique.

La durée du mandat présidentiel peut être affectée par une certaine interprétation de l'alinéa 2 de cet article 70 de la Constitution du 18 février 2006. En effet, il s'est dégagé des débats télévisés des « analystes politiques et juridiques » une thèse qui vise à porter atteinte à la durée du mandat présidentiel. C'est la stratégie du «glissement ». Pour les tenants de cette thèse, un Président de la République qui est arrivé à la fin de son mandat reste en fonction jusqu'à l'installation du nouveau Président de la République élu. Ce qui implique qu'un Président de la République peut rester au pouvoir aussi longtemps que possible tant qu'il n'y aura pas de nouveau Président de la République élu qui soit effectivement à même d'être installé.

Une telle interprétation est tout aussi erronée car étant faite en marge des techniques d'interprétation constitutionnelle. L'alinéa 2 de l'article 70 est à interpréter de manière holistique en le combinant avec l'article 73 de la Constitution. Aux termes de l'article 73, l'élection du Président de la République est organisée par la CENI, quatre-vingt-dix jours avant l'expiration du mandat du Président de la République en exercice. En effet, toute élection présidentielle doit être organisée au cours de trois mois avant la fin du mandat du Président en fonction. C'est pendant ce laps de temps que le Président en fonction reste au pouvoir jusqu'à la prise de fonction par son successeur à travers une cérémonie de prestation de serment présidentiel précédée par une séance de remise et reprise. Et ce laps de temps de trois mois fait partie de la durée du mandat présidentiel qui est de cinq ans. En d'autres termes, l'élection du nouveau Président de la République est organisée pendant que le mandat du Président précédent est en cours. La passation de pouvoir ou l'investiture du

27 Lumingu J.C., Evariste Boshab livre la raison de la révision constitutionnelle, L'Etendard des Révélateurs, $\mathrm{n}^{\circ} 00$ du 31 janvier au 6 février 2011, p. $3:$ «...le pouvoir que nous détenons, nous a été légué par Mzee Laurent Désiré KABILA, nous devons à tout prix, le conserver. Députés et Sénateurs ont fait leur part, il reste le vôtre et vous savez ce que vous devez faire...». 
nouveau Président doit avoir lieu au dernier jour du mandat du Président sortant, soit le dernier jour de la cinquième année de son mandat.

Dans ce sens, un Président de la République qui a fini son mandat (le premier ou le deuxième) ne peut rester au pouvoir même pour un seul jour, sauf l'hypothèse des circonstances exceptionnelles qui peuvent justifier la non tenue de l'élection présidentielle ou l'installation effective du Président de la République. Les seules circonstances exceptionnelles connues sont limitativement énumérées par la Constitution. Il s'agit de l'état de siège et de l'état d'urgence. ${ }^{28}$ Ainsi définies, les défis techniques, matériels et financiers relatifs à l'organisation d'une élection présidentielle ne peuvent pas être considérés comme de circonstances exceptionnelles.

Quant à l'article 220, il est éminemment protecteur de la durée et du nombre des mandats présidentiels. En effet, l'alinéa premier de cet article dispose :

La réforme républicaine de l'Etat, le principe du suffrage universel, la forme représentative du Gouvernement, le nombre et la durée des mandats du Président de la République, l'indépendance du pouvoir judiciaire, le pluralisme politique et syndical, ne peuvent faire l'objet d'aucune révision constitutionnelle.

La rédaction de cet alinéa ne souffre d'aucune ambiguïté et n'appelle même pas des techniques juridiques particulières pour saisir son contenu. Malgré cette nette clarté de l'article 220, une poignée de constitutionnalistes congolais opposent à l'intangibilité de cette importante disposition constitutionnelle, la théorie de la " double révision constitutionnelle ». Celle-ci consisterait à réviser un article « verrouillé » avant de modifier les matières qu'il contient. Pour eux, cet article 220 n'est pas lui-même verrouillé, donc il est susceptible de révision. Cette théorie est visiblement concoctée non pas pour le développement de la science juridique mais surtout en appui à une volonté politique de maintenir un individu au pouvoir en cherchant à « rendre ce qui est juridiquement impossible possible ». ${ }^{29}$

Une telle théorie, même dans l'hypothèse où elle serait rationnellement élaborée, est contraire à la lettre et à l'esprit de l'article 220. Cet article est insusceptible de révision. Cette affirmation découle d'une interprétation juridique rigoureuse. A la lecture de l'article 218 , il ressort qu'il n'y a que deux procédures consacrées pour réviser la Constitution du 18 février 2006. Il s'agit principalement de la voie du référendum ${ }^{30}$ et exceptionnellement par

28 Art. 85 de la Constitution dispose : «Lorsque des circonstances graves menacent, d'une manière immédiate, l'indépendance ou l'intégrité du territoire national ou qu'elles provoquent l'interruption du fonctionnement régulier des institutions, le Président de la République proclame l'état d'urgence ou l'état de siège, après concertation avec le Premier ministre et les Présidents des deux Chambres, conformément aux articles 144 et 145 de la présente Constitution. Les modalités d'application de l'état d'urgence et de l'état de siège sont déterminées par la loi ».

29 Propos de Kinkiey Mulumba, Ministre des Postes, Téléphones et Télécommunications et Président du Parti pour l'Action (PA) : «Je demande aux juristes de rendre possible ce qui parait impossible ». Verbatim de la Réunion de Kingakati (nom de la ferme du Président Joseph Kabila dans la périphérie de Kinshasa) du 25 août 2014.

30 Art. 218 al. 3 de la Constitution du 18 février 2006...op. cit. 
le Congrès parlementaire. ${ }^{31}$ Donc, lorsque l'on évoque la révision de la Constitution suivant l'ordre juridique actuel, elle ne revêt que ces deux modalités qui peuvent s'appliquer à tous les articles de la Constitution, sauf à l'article 220 et à ses correspondants. ${ }^{32}$

L'alinéa 1 de l'article 220, in fine, dispose que les matières qu'il énumère, c'est-à-dire la forme républicaine de l'Etat, le principe du suffrage universel, la forme représentative du Gouvernement, le nombre et la durée des mandats du Président de la République, l'indépendance du pouvoir judiciaire, le pluralisme politique et syndical, « ne peuvent faire l'objet d'aucune révision constitutionnelle ». En d'autres termes, aucune révision ne peut concerner ces matières. Aucune révision constitutionnelle voudrait dire ni la révision constitutionnelle par référendum ni celle par voie du Congrès parlementaire ; ni l'une ni l'autre; toutes les deux sont exclues. En logique, « aucun » est un quantificateur universel négatif qui exclut tout contrairement à « tout» qui est un quantificateur universel positif incluant tout. L'argument d'une révision constitutionnelle par référendum est inopérant et donc caduc.

A l'alinéa 2 de l'article 220, le constituant originaire s'exprime dans le sens du quantificateur universel positif pour le même objectif. Il a interdit, et de manière formelle, « toute révision constitutionnelle ayant pour objet ou pour effet de réduire les droits et libertés de la personne ou de réduire les prérogatives des provinces et des entités territoriales décentralisées ». Ainsi donc, les articles 3, 11 à 61, 197, 198, 203 et 204 ne peuvent pas être révisés ni par référendum ni par le Congrès parlementaire. Les groupes de mots « Est formellement interdite toute révision constitutionnelle... », voudrait dire que les deux formes de révisions constitutionnelles, "révision par référendum » et « révision par le Congrès parlementaire ", l'une et l'autre comprises, sont formellement interdites sur les matières relatives aux droits et les libertés de la personne ainsi que les prérogatives reconnues aux provinces et aux ETD.

Le débat dans la doctrine congolaise est très intense sur les matières constitutionnelles dites intangibles. Il devient cependant confus lorsque un certain nombre d'idéologues se font passer pour des scientifiques produisant des idées objectives, neutres et au service de l'humanité. Malheureusement, ceux qui ont été à l'avant-plan dans ce débat sont des « chercheurs du Gouvernement $»^{33}$, donc des idéologues rompus à la production des connaissances au service du pouvoir. ${ }^{34}$

31 Art. 218 al. 4 de la Constitution du 18 février 2006...op. cit.

32 L'article 220 de la Constitution est au sommet d'un réseau (sous-système) d'articles intangibles de manière expresse ou non. Il s'agit notamment des articles $1,3,5,6,7,8,90,11$ à 61, 64, 70, 197, 198,203 et 204.

33 Mbata Betukumesu Mangu A., Mandats présidentiels et révisions constitutionnelles en Afrique. La République Démocratique du Congo dans la perspective 2016, Kinshasa, 2013.

34 Sall E., CODESRIA Acceptance Speech, CODESRIA Bulletin, Numbers 3 \& 4, 2013, p. 3. 
Jean-Louis Esambo affirme que

...mêmes intangibles, les dispositions constitutionnelles peuvent être modifiées à condition, d'une part, de respecter la procédure de révision et, d'autre part, d'obtenir que les motifs de révision ne soient pas équivoques et emportent l'adhésion de la majorité de citoyens intéressés. ${ }^{35}$

C'est ici le lieu de lui poser la question de savoir la procédure à respecter pour la révision des dispositions intangibles lorsque l'on sait que celles-ci ont été mises hors de toutes les deux formes de révisions prévues à l'article 218 de la Constitution. Pensait-il trouver quelques motifs univoques, à l'époque où il rédigeait son ouvrage, pour justifier la révision des dispositions intangibles? Dans l'hypothèse où l'adhésion de la majorité des Congolais serait obtenue pour modifier les articles intangibles, l'on parlerait toujours de la révision constitutionnelle?

La position de Jean-Louis Esambo reflète la tendance, minoritaire par ailleurs, de la doctrine congolaise qui pense que les dispositions intangibles posent un problème juridique sans le circonscrire et le démontrer. L'interprétation de ces dispositions est faite à l'aune de la doctrine européenne ; loin du contexte historique et politique congolais de leur émergence et du droit comparé africain. Félix Vunduawe te Pemako et Jean-Marie Mboko Dj'Andima écrivent ce qui suit:

Sans rejeter l'idée selon laquelle la révision constitutionnelle doit malgré tout parvenir à un équilibre raisonnable entre souci d'adapter la Constitution à de nouvelles réalités et celui de préserver son identité, il est permis de reconnaître que la limitation du pouvoir de révision en raison de la matière pose problème, car elle introduit l'idée d'une supra-constitutionnalité de certains droits, principes ou règles dans la Constitution. ${ }^{36}$

A l'appui de leur thèse, une panoplie de citations d'auteurs européens. Il importe de les mentionner avec leurs idées dans cette étude : Francis Delpérée qui soutient que « ..., formellement rien ne distingue les dispositions constitutionnelles les unes des autres..., le constituant originaire ne saurait lier à perpétuité le titulaire de la souveraineté, le peuple ». Georges Vedel qui écrit que «...le pouvoir constituant dérivé n'est pas un pouvoir d'une autre nature que le pouvoir initial : la Constitution lui donne sa procédure..., elle ne borne point son étendue ». Pierre Pactet qui a soutenu qu' " on est obligé d'admettre que si ces interdictions sont transgressées, il n'y a pratiquement aucune sanction possible, parce que le plus souvent la Constitution ne prévoit pas de recours juridique contre la loi de révision ». Pour Elisabeth Zoller, «c'est admettre que les gardiens de la Constitution, et en particulier les juges, sont au-dessus du pouvoir constituant, c'est-à-dire le peuple ». Georges Vedel

35 Esambo Kangashe J.L., Le droit constitutionnel, Bruxelles, 2013, p. 103.

36 Vunduawe te Pemako F. et Mboko Dj'Andima J.M., Droit constitutionnel du Congo. Textes et documents fondamentaux, Vol. 2, Bruxelles, 2013, p. 1073. 
renchérit : " le pouvoir constituant étant le pouvoir suprême de l'Etat, (il) ne peut être lié, même par lui-même ». Charles Debbasch pense, quant à lui, qu' "une telle limitation peut même aboutir à imposer un recours au renversement de l'ordre constitutionnel par la force d'une révolution $»{ }^{37}$

Cette liste d'auteurs cités par Félix Vunduawe et Jean-Marie Mboko à l'appui de leur thèse n'est pas exhaustive. Elle le serait si elle avait inclut d'autres auteurs notamment français qui trouvent en ce que d'aucuns n'ont pas hésité d'appeler « clauses d'éternité » ${ }^{38}$, l'émergence d'un droit constitutionnel fondamental. ${ }^{39}$

Les deux auteurs concluent que

Les conditions d'adoption d'une révision constitutionnelle sous l'empire de la Constitution congolaise actuelle confortent d'ailleurs l'idée selon laquelle il n'existe pas de principes supra-constitutionnels. Car la révision constitutionnelle peut être approuvée par référendum, c'est-à-dire par le peuple lui-même, titulaire de la souveraineté. Si c'est le souverain lui-même qui adopte la révision, on ne voit pas comment on pourrait prétendre sérieusement lui imposer le respect de ses volontés antérieures. ${ }^{40}$

Cette affirmation ressemble à ce que Léon de Saint Moulin appelait déjà « ...un exercice académique dans un pays indéterminé $»{ }^{41}$ L'interprétation de l'article 220 telle que proposée par Félix Vunduawe et Jean-Marie Mboko est faite du point de vue étranger sans tenir compte des réalités congolaises. Le peuple peut se limiter. Cette limitation implique le respect par lui-même de ses volontés antérieures. Rien n'interdit que le peuple s'impose le respect de ses propres volontés lorsque les circonstances qui avaient justifié leur consécration n'ont pas changé.

Un autre exemple d'interprétation de la Constitution congolaise du point de vue européen est donné par le fait que ces deux auteurs n'ont pas trouvé d'inconvénients dans la révision de l'alinéa 2 de l'article 149 de la Constitution qui exclue les parquets du cercle restreint des détenteurs du pouvoir judiciaire. ${ }^{42}$ Selon eux, « ...la révision constitutionnelle du 20 janvier 2011 a clarifié les choses... ». ${ }^{43}$ Un point de vue qui n'est pas partagé par

37 Vunduawe te Pemako F. et Mboko Dj'Andima J.M.,...op. cit., pp. 1073-1074.

38 Voir Boshab Mabuj E., Entre la révision constitutionnelle et l'inanition de la nation, Bruxelles, 2013.

39 Voir Favoreu L. et al., Droit constitutionnel, Paris, 2011, pp. 117-119.

40 Vunduawe te Pemako F. et Mboko Dj'Andima J.M.,...op. cit., p. 1074.

41 de Saint Moulain L., Projet de Constitution de la RDC. Dimension sociale, Congo-Afrique, $\mathrm{n}^{\circ} 397$, septembre 2005, pp. 84-94.

42 Alinéa 2 de l'article 149 de la Constitution : « Il (pouvoir judiciaire) est dévolu aux Cours et Tribunaux qui sont : la Cour constitutionnelle, la Cour de cassation, le Conseil d'Etat, la Haute Cour militaire, les cours et tribunaux civils et militaires ainsi que les parquets rattachés à ces juridictions ").

43 Vunduawe te Pemako F. et Mboko Dj'Andima J.M.,...op. cit., p. 1049. 
d'autres juristes congolais. En tout état de cause, le peuple congolais n'avait commis aucune faute en incluant les parquets parmi les titulaires du pouvoir judiciaire et les rendant indépendants des pouvoirs exécutif et législatif.

C'est dans ce sens qu'abondent Pierre Akele Adau et Auguste Mampuya Kanunk'a Tshiabo. Suivant Pierre Akele Adau,

En réalité, la logique de l'ancienne formulation de cette disposition constitutionnelle entendait tirer conséquence de la nature hybride de l'officier du ministère public en le faisant participer pleinement à l'expression de l'indépendance du pouvoir judiciaire lorsqu'il agissait en qualité de magistrat. Ceci devait amener un réaménagement législatif des rapports entre le parquet et le pouvoir exécutif; mais c'eût été pour le gouvernement accepter de prendre le risque de perdre le contrôle du parquet, maillon essentiel de son influence ou de ses interférences sur le pouvoir judiciaire. De sorte que l'on peut se demander si cet amendement-là n'a pas été le premier essai réussi du contournement du 'fameux" article 220 qui continue à affirmer que l'indépendance du pouvoir judiciaire ne peut faire l'objet d'aucune révision, et donc un cas patent de haute trahison par la violation intentionnelle de la Constitution, ... ${ }^{44}$

Beaucoup plus explicite, Auguste Mampuya Kanunk'a Tshiabo fait observer que la révision de l'article 149 est à la fois une atteinte à l'article 220 et un recul. Il écrit que :

Notre article 149 était rédigé dans une logique implacable : dans l'alinéa $1^{\text {er }}$ il affirmait l'indépendance du pouvoir judiciaire; dans l'alinéa 2, sans doute pour la première fois dans l'histoire constitutionnelle, il définissait avec bonheur ce pouvoir judiciaire comme comprenant " les cours et tribunaux : la Cour constitutionnelle, la Cour de cassation, le Conseil d'Etat, la Haute Cour militaire, les cours et tribunaux civils et militaires ainsi que les parquets rattachés à ces juridictions. ${ }^{45}$

Auguste Mampuya constate que la révision constitutionnelle de l'article 149 a supprimé

... le pouvoir judiciaire comme corps notamment dans la disposition de l'alinéa 2 de l'art. 149 d'où disparaît l'heureuse définition du pouvoir judiciaire de nouveau limité aux seuls cours et tribunaux sans les parquets. La mauvaise intention était telle que, dans la proposition initiale, disparaissait de même l'alinéa $1^{\text {er }}$ qui dit que «le pouvoir judiciaire est indépendant du pouvoir législatif et du pouvoir exécutif », donc l'énoncé essentiel même du principe de l'indépendance. ${ }^{46}$

44 Akele Adau P., Réponses pénales au discours du désordre ou au désordre du discours constitutionnel en République Démocratique du Congo : la Cour constitutionnelle à l'épreuve, Revue africaine de la démocratie et de la gouvernance, Vol. I, n 2 \& 3, 2014, p. 49 (pp. 43-62).

45 Mampuya Kanunk'a-Tshiabo A., Le respect des textes légaux comme problème d'éthique de gouvernance au Congo, Revue de l'Université du Kasayi, Vol. 1, n 1, Février 2013, p. 153. (pp. 145-165).

46 Mampuya Kanunk'a-Tshiabo A., op. cit., pp. 153-154. 
Selon lui, l'alinéa 2 de l'article 149 dans sa version originaire était une forte avancée de l'Etat de droit en RDC par rapport à certaines vieilles démocraties occidentales. En étendant le bénéfice de l'indépendance du pouvoir judiciaire jusqu'aux parquets, le constituant de 2006 rejetait la fameuse « règle classique ${ }^{47}$

en faveur de la conception moderne que l'on trouve dans les pays anglo-saxons et que la France que nous aimons bien copier quand il s'agit de mauvais choix, est en train de mettre en place, sous le coup de condamnations répétées des instances internationales refusant de considérer ses procureurs comme " autorités judiciaires 》 et les considérant comme des organes non indépendants. ${ }^{48}$

Il conclut que

Dans la conception moderne, l'indépendance du pouvoir judiciaire concerne également le ministère public; en tout état de cause, ce fut l'option, innovante et révolutionnaire, du constituant congolais au regard de ce que les auteurs appellent " règle classique " et qui ne l'est plus dans bien des pays, réviser cette disposition, comme l'a fait la révision, va bien à l'encontre de la lettre et de l'esprit de la constitution en son article 149 sur l'indépendance de la justice, contrairement à l'interdiction de l'art. $220 .{ }^{49}$

Au sujet du principe de supra-constitutionnalité, la supériorité de l'article 220 sur les autres articles de la Constitution est non seulement attestée dans l'opinion publique, à travers les pétitions contre sa révision mais aussi à travers des discours politiques ou de prises de position de leaders sociaux. Même les partisans de la révision constitutionnelle se sont ravisés en recourant au concept de « changement de Constitution ». Ainsi dans son discours à l'ouverture de la session parlementaire du mois de septembre 2014, le Président du Sénat Léon Kengo wa Dondo a déclaré que les dispositions constitutionnelles intangibles constituent « le socle de toute l'architecture constitutionnelle ». De leur côté, les évêques catholiques ont estimé que « ces dispositions posent les bases de la stabilité du pays et de l'équilibre des pouvoirs dans les institutions $» .{ }^{50}$ Une question qui peut tarauder l'esprit de tout observateur averti est celle de savoir si un principe consacré dans la Constitution peut être toujours considéré comme supra-constitutionnel.

Une autre hypothèse qui pourrait porter atteinte à l'intangibilité du principe de limitation du nombre et de la durée des mandats présidentiels est le prolongement de fait d'un mandat présidentiel. C'est ce que l'on appelle dans le langage politique congolais « glissement » analysé ci-dessous.

47 Qui voudrait que le parquet exerce son ministère sous l'autorité du ministre de la justice.

48 Mampuya Kanunk'a-Tshiabo A., op. cit., p. 154.

49 Mampuya Kanunk'a-Tshiabo A., op. cit., p. 155.

50 Radio Okapi, Révision de la constitution : faut-il toucher les articles irréformables?, http://radiook api.net/emissions-2/ (Consulté le 30 avril 2015). 


\section{Le statut d'ancien Président de la République}

Le statut d'ancien Président de la République est une conséquence directe de l'alternance au pouvoir. Elle est conçue à la fois comme un devoir de l'Etat envers les citoyens loyaux ayant présidé à sa destinée et une garantie pour assurer une alternance politique et démocratique dans l'Etat. Dans le premier cas, un ancien Président de la République a droit à la protection après son départ du pouvoir. Cette protection doit être à la fois politique, économique et sociale pour lui permettre d'être à l'abri de tout ce qui peut « souiller » la fonction qu'il a incarné à un moment donné de l'histoire de toute la nation. Cette protection est traduite par des avantages lui accordés et dont l'objectif est de le rassurer de l'existence d'une « vie après la Présidence ».

Le constituant du 18 février 2006 était bien conscient de cette réalité. Ce qui prouve qu'il tenait mordicus au principe cardinal de l'alternance politique démocratique au pouvoir en RDC. C'est ce qu'il a fait en instituant un statut pour les anciens Présidents de la République élus. En effet, l'alinéa 6 de l'article 104 de la Constitution dispose : « Les anciens Présidents de la République élus sont de droit sénateurs à vie ». En d'autres termes, un ancien Président de la République élu est, dès son départ du pouvoir, sénateur pour toute la durée de sa vie sur cette terre des hommes. Ce statut de sénateur à vie n'est pas reconnu aux anciens Présidents de la République non élus. Ceux-ci sont des anciens Présidents de la République intérimaires.

A quels avantages le statut de sénateur à vie renvoie-t-il ? Un sénateur à vie jouit de tous les droits que tout autre sénateur. Il est bénéficiaire des immunités judiciaires ${ }^{51}$ et à droit à une indemnité équitable qui lui permet d'assurer son indépendance et sa dignité. ${ }^{52}$ Au sujet des immunités judiciaires, le constituant du 18 février 2006 a mis en évidence le principe de lutte contre l'impunité surtout pour les crimes les plus graves. Le bénéfice des immunités judiciaires ne signifient pas impunité. Ces immunités peuvent être levées pour permettre à un ancien Président de la République de répondre de ses actes infractionnels.

En instituant les immunités pour les parlementaires, le constituant n'a nullement eu l'intention de les préserver des poursuites judiciaires. Sa volonté de lutter contre l'impunité est maximale. Le constituant du 18 février 2006 est beaucoup plus précis en ce qui concerne les infractions commises en dehors de l'exercice de fonctions présidentielles. En effet, un ancien Président de la République peut être poursuivi pour des infractions commises par lui et qui n'avaient rien avoir avec l'exercice des fonctions présidentielles. ${ }^{53} \mathrm{La}$ Constitution est muette au sujet de la dénomination du juge chargée de connaître de ces infractions. Peut-on aussi admettre que son silence renvoie également au droit commun ?

La question qui se pose à ce stade de réflexion est celle de savoir si ces avantages accordés aux anciens Présidents de la République suffisent-ils pour persuader un Président de

51 Art. 107 de la Constitution du 18 février 2006...op. cit.

52 Art. 109 de la Constitution du 18 février 2006...op. cit.

53 Art. 167 de la Constitution du 18 février 2006...op. cit. 
la République en exercice à respecter le principe de l'alternance politique et à transmettre le pouvoir à son successeur? La réponse est négative au regard des opinions émises par des acteurs politiques et sociaux en RDC.

Il ressort des débats engagés par les acteurs politiques et sociaux congolais que le statut des anciens Présidents de la République élus tel que défini par la Constitution n'est pas suffisant pour dissuader un Président de la République à se cramponner au pouvoir. D'où la nécessité des garanties supplémentaires. Celles-ci devaient être coulées dans une loi portant statut des anciens Présidents de la République. Les débats sur cette initiative ont quitté les plateaux de radios et télévisions kinoises pour emprunter le chemin parlementaire avec la proposition de loi portant statut des anciens Chefs de l'Etat initiée par le sénateur Modeste Mutinga Mutuishay, du Mouvement Social pour le Renouveau (MSR), un parti politique de la Majorité présidentielle (MP).

Quel devra être le contenu et les limites de cette loi ? La question est très débattue dans les milieux des organisations de la Société civile. Pour elles, cette loi devra déterminer les droits supplémentaires pour les anciens Présidents de la République et leurs imposer un certain nombre d'obligations en contreparties. Dans le registre des droits, on dénombre les libertés civiles, politiques et économiques. Les anciens Présidents de la République devraient avoir droit à une pension mensuelle et à une garde rapprochée pour leur protection. Ils devraient aussi avoir droit aux privilèges protocolaires au cours de manifestations publiques officielles. Dans cette perspective, les anciens Présidents de la République peuvent accompagner le Président de la République en exercice à certaines cérémonies internationales pour défendre la cause du pays. Ils peuvent être délégués pour représenter l'Etat à ces cérémonies.

Au sujet des garanties judiciaires, il se dégage une unanimité au sein des Organisations de la Société civile qu'un ancien Président de la République ne peut faire l'objet de poursuites judiciaires intempestives. Toutefois, il doit répondre des actes d'enrichissement illicite par la restitution des biens et fonds indûment acquis et des crimes internationaux. La future loi sur le statut d'anciens Présidents de la République devrait comporter aussi des obligations à leur charge. La plus importante de ces obligations est l'interdiction d'interférence dans la gestion des affaires publiques.

Cette dernière obligation pèche par sa généralisation. L'on devrait faire un distinguo entre un ancien Chef de l'Etat ayant fini ses deux mandats et celui qui n'en a fait qu'un seul. Dans le premier cas, un tel ancien Président ne peut exercer des activités politiques visant à revenir au pouvoir. Dans le second, il est autorisé à un ancien Président qui n'avait fait qu'un seul mandat de prétendre à un deuxième mandat présidentiel. Dans cette perspective, ce dernier peut s'intéresser à la gestion de la chose publique par des critiques annonçant son nouveau programme politique.

Si malgré tous ces avantages, un Président de la République s'obstine à se maintenir au pouvoir au-delà de son ultime mandat, le constituant du 18 février 2006 a prévu un mécanisme très robuste et redoutable pour tout dirigeant récalcitrant. C'est le devoir imposé à 
tout congolais de faire échec à tout individu ou groupe d'individus qui prend le pouvoir par la force ou qui l'exerce en violation de la Constitution. ${ }^{54}$

III. Le devoir de résistance à la prise de pouvoir par la force ou à son exercice en violation de la Constitution

L'une des caractéristiques singulières de la Constitution du 18 février 2006 est la consécration du devoir de résistance à la prise de pouvoir par la force ou à son exercice en violation de la Constitution. Ce devoir élevé au rang des principes fondamentaux du nouvel ordre constitutionnel et politique congolais n'est pas une invention du constituant du 18 février 2006. Sa première occurrence remonte à 1992 sous la forme d'un droit de résistance consacré dans l'Acte portant dispositions constitutionnelles relatives à la période de transition adoptée par la Conférence nationale souveraine. Suivant l'article 37 alinéa 2 de cette Constitution de Transition,

Tout Zaïrois (Congolais) a le droit sacré de... désobéir et de résister à tout individu ou groupe d'individus qui prend le pouvoir par la force ou l'exerce en violation du présente Acte.

Cette disposition constitutionnelle a été reprise avec une certaine évolution à l'article 37 l'Acte constitutionnel de la transition (ACT) de la République du Zaïre du 09 avril 1994 en ces termes :

Tout Zaïrois a le droit et le devoir sacrés de défendre la Nation et son intégrité territoriale et de faire échec à tout individu qui prend le pouvoir par la force ou l'exerce en violation des dispositions du présent Acte. ${ }^{55}$

L'apport de l'ACT dans l'évolution du devoir de résistance à la prise de pouvoir par la force ou à son exercice en violation de la Constitution est le doublement de sa nature juridique. Il n'est plus seulement un devoir mais aussi un droit sacré pour tout citoyen. L'alinéa 2 de cette disposition fournit des détails tendant à expliquer l'exercice du pouvoir en violation de la Constitution. Le constituant de transition du 9 avril 1994 avait prescrit que :

Nul ne peut détourner les attributs du pouvoir et la puissance publique à des fins personnelles pour la réalisation d'intérêts partisans ou pour faciliter l'ingérence d'une institution ou d'un service public dans le fonctionnement d'une autre institution ou d'un autre service public.

Il importe de noter que la consécration du devoir-droit de résistance n'était pas seulement une préoccupation des Zaïrois. D'autres peuples d'Afrique avaient insérer des dispositions

54 Art. 64 al. 1 de la Constitution du 18 février 2006...op. cit.

55 Voir l'article 37 de l'Acte Constitutionnel de transition, J.O.Z, $35^{\text {ème }}$ année, numéro spécial du 9 avril 1994. 
pareilles dans leurs constitutions ; surtout celles consécutives aux Conférences nationales. ${ }^{56}$ Cette recette est la résultante du processus démocratisation de l'Afrique relancé au début des années 1990.

Cependant, le droit de la résistance date des siècles. En effet, la résistance a toujours été fondée sur le droit naturel considéré comme le droit rationnel tel que la raison humaine le découvre dans la conscience de chaque homme. ${ }^{57}$ A la base de cette théorie du droit naturel, subsiste l'idée qu'il existe au-dessus des autorités des règles qui s'imposent à elles et auxquelles elles doivent se conformer dans l'exercice de leur pouvoir. C'est sous cette forme que la théorie du droit naturel a inspiré la déclaration des droits de l'homme et du citoyen, et toute la pensée politique moderne en Occident. ${ }^{58} \mathrm{C}$ 'est ainsi que la déclaration des droits de l'homme et du citoyen de 1789 rangeait le droit de résistance à l'oppression parmi les droits naturels et imprescriptibles de l'homme. ${ }^{59}$

Tel qu'inscrit dans la Constitution du 18 février 2006, le droit de résistance est une garantie pour assurer l'alternance politique en RDC. Il constitue une pression sur tout Président de la République en fonction qui chercherait à s'y maintenir. Devant une volonté affichée de ne pas respecter le principe de l'alternance politique, le peuple congolais doit recourir à tous les moyens de droit pour faire échec à cette entreprise. Il peut user d'une gamme d'actions dont les pétitions, les manifestations publiques, les grèves générales, les villes mortes, la désobéissance civile, l'insurrection populaire ou la révolution. Toutes ces actions doivent viser la promotion de l'alternance politique au pouvoir en contraignant les tenants du pouvoir à le céder au terme de leur mandat ou à déloger ceux qui y sont parvenir par des voies inconstitutionnelles.

\section{Les atteintes et les tentatives d'atteintes au principe de l'alternance politique en RDC}

Les atteintes et les tentatives d'atteintes à l'alternance politique en RDC sont ressorties à partir du fonctionnement des institutions de la troisième République. En effet, toutes les institutions politiques prévues par la Constitution du 18 février 2006 ont été installées entre 6 décembre 2006 et 14 mai 2007 au niveau central. Le premier mandat pour le Président de la République a connu son terme le 6 décembre 2011 et le deuxième mandat et le dernier se termine le 20 décembre 2016. Si l'Assemblée nationale a été renouvelée au terme de cinq ans de première législature, le Sénat fonctionne au-delà de son premier mandat. Le non-respect des délais est une préfiguration des atteintes au principe de l'alternance politique.

56 Lire dans ce sens Ntumba Luaba, Le projet de la Constitution de la République du Zaïre, ZaïreAfrique, 1993, n³10, p. 538.

57 Duverger M., Institutions politiques et droit constitutionnel, $7^{7 \mathrm{eme}}$ édition, Paris, 1963, p. 54.

58 Idem.

59 Ellul J., Histoire des institutions politiques, Paris, 1956, p.10. 
I. Les atteintes au principe de l'alternance politique en RDC

Les atteintes à l'alternance politique en RDC ont été localisées dans une seule période. Il s'agit de l'année 2011 qui coöncidait avec la fin de la première mandature du Président de la République. Deux atteintes peuvent être répertoriées dans le cadre de cette étude. Il s'agit de la révision de la Constitution du 18 février 2006 et de la fraude électorale.

a. La révision constitutionnelle du 20 janvier 2011

La Constitution du 18 février 2006 a connu sa toute première révision au début de sa cinquième année d'existence. Cette révision n'a concerné que huit articles. Seul l'article 71 sur les huit intéresse cette réflexion et portait du coup atteinte au principe de l'alternance au pouvoir. En effet, l'article 71 appartient au troisième titre de la Constitution relatif à l'organisation et à l'exercice du pouvoir en RDC. Au sein de ce titre, l'article 71 fait corps avec les articles touchant à l'institution "Président de la République ». Cet article consacre l'élection présidentielle à la majorité absolue des suffrages exprimés. Au cas où cette majorité ne serait pas obtenue au premier tour de l'élection présidentielle, il devait être organisé un second tour du scrutin présidentiel. ${ }^{60}$

Le deuxième tour de l'élection présidentielle était organisée uniquement pour les deux candidats ayant recueilli le grand nombre des suffrages exprimés au premier tour. Il ne s'agit ici que de deux premiers candidats ayant obtenu beaucoup de voix. ${ }^{61}$ Lorsque, dans l'entre-deux tours, un des candidats décède, tombe dans un cas d'empêchement ou désiste, les candidats malheureux du premier tour sont repêchés suivant l'ordre d'arrivée du premier tour de l'élection présidentielle. ${ }^{62}$

A l'issue du second tour de l'élection présidentielle, est déclaré élu le candidat ayant recueilli la majorité des suffrages exprimés. ${ }^{63} \mathrm{Tel}$ est le contenu de l'article 71 originaire. Tel qu'il a été rédigé, cet article était clair et ne souffrait d'aucune ambiguïté de nature à rendre son application difficile. En dépit de cette clarté, l'article 71 est le premier à être visé par la révision du 20 janvier 2011. En quoi a consisté cette révision?

L’article 71 dérivé est libellé comme suit : «Le Président de la République est élu à la majorité simple des suffrages exprimés ». Les trois alinéas de l'article 71 originaire ont été supprimés. Ils concernaient le second tour de l'élection présidentielle. La nouveauté appor-

60 Art. 71 al. 1 de la Constitution de la RDC, JORDC, $47^{\text {ème }}$ année, numéro spécial du 18 février 2006 dispose : «Le Président de la République est élu à la majorité absolue des suffrages exprimés. Si celle-ci n'est pas obtenue au premier tour du scrutin, il est procédé, dans un délai de quinze jours, à un second tour ».

61 Art. 71 al. 2 de la Constitution...op. cit. dispose : «Seuls peuvent se présenter au second tour, les deux candidats qui ont recueilli le plus grand nombre des suffrages exprimés au premier tour ».

62 Art. 71 al. 3 de la Constitution...op. cit.

63 Art. 71 al. 4 de la Constitution...op. cit. 
tée par la révision de cet article est l'élection du Président de la République à une majorité simple en lieu et place de la majorité absolue de l'article 71 ancien.

La révision de cet article pendant l'année électorale et au terme du mandat du Président de la République sortant était perçue comme une astuce constitutionnelle de s'assurer une réélection dans un contexte où le champ politique et le climat social n'était plus favorable au régime en place. De ce fait, la révision de l'article 71 était une atteinte au principe de l'alternance politique en RDC. Au-delà de la révision constitutionnelle du 20 janvier 2011, la fraude à l'élection présidentielle du 28 novembre de la même année constituait une deuxième atteinte à l'alternance politique en RDC. ${ }^{64}$

\section{b. La fraude à l'élection présidentielle du 28 novembre 2011}

La fraude électorale est une atteinte au principe de l'alternance politique dans la mesure où le Président de la République sortant conserve le pouvoir en étant proclamé élu au lieu du candidat qui a été réellement élu. La question qu'il convient de se poser ici est celle de savoir si la fraude à l'élection présidentielle du 28 novembre 2011 a porté atteinte à l'alternance politique en RDC. La majorité d'acteurs qui sont intervenus dans l'organisation et l'observation de l'élection présidentielle du 28 novembre 2011 étaient unanimes sur le fait que cette élection a été émaillée des irrégularités. La seule différence n'a résidé que sur l'impact de ces irrégularités sur l'ordre de classement des candidats à cette élection présidentielle.

Pour les partisans du candidat ayant emporté cette élection présidentielle, les irrégularités ayant entachées le déroulement de l'élection présidentielle n'étaient pas de nature à inverser l'ordre de classement des candidats. Pour les candidats opposants, les résultats de l'élection présidentielle publiés par la CENI le 9 décembre 2011 n'étaient pas conformes aux votes exprimés dans les urnes. C'est la même proposition soutenue par la Société civile $^{65}$ et les observateurs internationaux ${ }^{66}$, à l'exception de ceux de l'Union africaine, de la SADC, de la CEEAC et du COMESA.

64 Lire dans ce sens Akele Adau P., Réponses pénales au discours du désordre constitutionnel...op. cit., p. 51.

65 AETA, Lettre de la Société civile congolaise au Secrétaire général des Nations Unies sur la crise post-électorale en RDC, Kinshasa, le 07 février 2012. Mission nationale d'observation, Rapport à mi-parcours de l'observation des élections du 28 novembre 2011, Kinshasa, le 17 décembre 2011; Conférence Episcopale Nationale du Congo, Le peuple congolais a faim et soif de justice et de paix. Le courage de la vérité (cf. 2 Co 7, 14). Message de l'Assemblée plénière extraordinaire de la CENCO aux fidèles catholiques et à l'ensemble du peuple congolais, Kinshasa, Editions du Secrétariat général de la CENCO, 2012, par. 8.; Ngapi R., En soutien à la déclaration du Cardinal Monsengwo. Le clergé catholique kinois : 'La vérité vous rendra libres', Le Potentiel, $n^{\circ} 5407$ du Lundi 19 décembre 2011. Le Potentiel, Résultats des élections 2011. Monsengwo jette de l'huile sur le feu, Le Potentiel, n 5402 du mardi 13 décembre 2011.

66 Mission d'observation électorale de l'Union européenne en République Démocratique du Congo, Rapport final des élections présidentielle et législatives du 28 novembre 2011, www.moeue-rdc.eu (Consulté le 21 novembre 2011). 
En tout état de cause, les résultats de l'élection présidentielle publiés par la CENI ne reflétaient pas la volonté du peuple. Dans ce contexte de cafouillage, le vainqueur réel de l'élection présidentielle n'a pas été connu. Le rôle joué par la CENI et la CSJ n'a pas non plus promu l'alternance politique en $2011 .^{67}$

\section{Les tentatives d'atteinte au principe de l'alternance politique en RDC}

Les tentatives d'atteintes au principe de l'alternance politique sont situées essentiellement à partir de l'année 2013. Le fait révélateur reste la publication par Evariste Boshab de l'ouvrage intitulé « Entre la révision constitutionnelle et l'inanition de la Nation » paru en juin 2013. L'auteur de l'ouvrage soutient la thèse selon laquelle tous les articles de la Constitution, même ceux déclarés intangibles, sont révisables. La présentation de cet ouvrage a eu l'avantage de lancer les débats sur la révision constitutionnelle.

\section{Les tentatives de révision constitutionnelle}

Au cours de sa $23^{\text {ème }}$ réunion extraordinaire tenue le 9 juin 2014, le Conseil des ministres a adopté quatre projets de lois soumis par le Ministre de l'intérieur dont celui portant révision de certains articles de la Constitution telle que modifiée par la Loi ${ }^{\circ} 11 / 002$ du 20 janvier 2011 portant révision de certains articles de la Constitution de la République Démocratique du Congo ». Ce projet de loi visait la modification de quatre articles de la Constitution. Il s'agit des articles 101, 104, 197 et 198. Sur le plan du contenu, le projet de loi de révision de la Constitution prévoyait la scission de la loi électorale en quatre lois spécifiques et le changement de mode d'élection des députés provinciaux de l'élection au suffrage universel direct au suffrage universel indirect.

Le projet de loi visant la révision de la Constitution déposé au Parlement n'a cependant concerné que la révision de l'article 197 de la Constitution, plus particulièrement l'élection des députés provinciaux au suffrage universel indirect par les conseillers communaux. Comme indiqué ci-haut, cet article est verrouillé par l'article 220 qui interdit toute révision constitutionnelle tendant à réduire les droits et les libertés fondamentaux des citoyens congolais. Changer le mode d'élection des députés provinciaux en le ramenant à l'élection indirecte, revient à priver les citoyens congolais de leur droit de voter leurs députés provinciaux.

La révision de l'article 197 ne concerne pas l'alternance politique. Mais beaucoup d'observateurs ont vu en cette tentative une manière d'atteindre indirectement l'article 220 pour le ruiner petit à petit avant qu'il ne soit amputé purement et simplement de la Constitution. Le projet de loi portant révision de la Constitution a été retiré du Parlement par le Gouvernement dans la foulée de la révolution populaire au Burkina Faso (du 30 au 31 octobre 2014). Cette révolution qui a abouti au renversement du régime en place à la suite de la

67 C.S.J, 16 décembre 2011, RCE 011/PR, inédit. 
volonté obstinée du Président Blaise Compaoré de réviser la Constitution afin de se maintenir au pouvoir après 27 ans de règne sans partage. ${ }^{68}$

2. L'hypothèse d'une prolongation de fait du mandat présidentiel ou stratégie du glissement

La prolongation de fait du mandat présidentiel est une situation de fait accompli résultant de l'absence d'organisation des élections à des échéances prévues. Elle est perçue, par les opposants et les activistes de la Société civile, comme un moyen ultime pour les tenants du régime pour amorcer une transition politique qui remettrait le compteur de mandats présidentiels à zéro.

Contre toute idée de prolongation d'un mandat présidentiel, il faut considérer la fin d'un mandat présidentiel sans qu'il y ait élection du nouveau Président de la République comme une cause de vacance à la Présidence de la République. Si jusqu'au 17 décembre 2016, le nom du nouveau Président de la République n'est pas connu, la Cour constitutionnelle devra être obligée de déclarer la vacance à la Présidence de la République. Les fonctions du Président de la République seront assumées par le Président du Sénat ${ }^{69}$ pour une durée qui ne peut dépasser cent vingt jours quelles que soient les circonstances.

Dans le contexte actuel de la RDC, des difficultés peuvent poindre à l'horizon dans l'application de cette disposition suivant l'interprétation que je viens de donner. L'article 76 de la Constitution exige que la Cour constitutionnelle soit saisie par le Gouvernement en cas de vacance à la Présidence de la République. Or, l'actuel Gouvernement est acquis entièrement au Président de la République qui trouverait intérêt dans le glissement de son ultime mandat. Il parait donc impossible, sauf retournement de tendance, à ce Gouvernement de saisir la Cour constitutionnelle pour déclarer la vacance à la Présidence de la République. Ce qui est une autre façon de contraindre le Président de la République à partir contre son gré.

Dans l'hypothèse où la Cour constitutionnelle serait saisie, là encore, beaucoup de doutes persistent qu'elle dise le bon droit. Dans sa configuration actuelle, la Cour constitutionnelle n'est pas différente de la défunte Cour suprême de Justice (CSJ) qui, sur les questions de constitutionnalité qui lui avaient été soumises, elle s'était comportée comme un bras séculier du Président de la République et de ses partisans en coulant les volontés poli-

Justice pour Thomas Sankara, Justice pour l'Afrique, Blaise Compaoré chassé par son peuple ! Il ne doit pas échapper à la justice, http://pambazuka.org/fr/category/features/93302, (Consulté le 03 janvier 2015) ; Aziz Salmone Fall, Burkina, vers la libération, http://pambazuka.org/fr/category/ features/93299, (Consulté le 03 janvier 2015), Bruno Jaffré, Burkina Faso : Blaise Compaoré vient de déclarer la guerre à son peuple, http://pambazuka.org/fr/category/features/93300, (Consulté le 03 janvier 2015).

69 Art. 75 de la Constitution du 18 février 2006...op. cit, cite comme cas d'ouverture de la vacance au pouvoir, le décès, la démission ou « toute autre cause d'empêchement définitif ». L'expression « toute autre cause » imprime un caractère illimité de causes d'interruption d'un mandat présidentiel. 
tiques en des arrêts de justice, insusceptibles de recours et opposables à tous. Sur les 9 membres de cette Cour, 4 sont des anciens juges de la triste CSJ et étaient impliqués dans l'examen des contentieux de l'élection présidentielle tant de 2006 que de $2011^{70}$. Les 4 professeurs d'Université qui font partie de la composition de cette Cour sont en majorité des militants du parti présidentiel (Parti du Peuple pour la Reconstruction et la Démocratie, PPRD). Une telle composition partisane de la Cour constitutionnelle ne trouverait pas d'inconvénients, même sans soubassement juridique, de prononcer un glissement du mandat présidentiel.

Cependant, l'opinion publique congolaise est bien informée sur cette question précise. La prise de position des organisations de la Société civile est beaucoup plus claire. Pour elles, il ne saurait y avoir une prolongation du mandat présidentiel à cause d'un report des élections. La Société civile a mis en garde la majorité présidentielle qui pourrait être tentée de se servir du précédent malheureux de la prolongation inconstitutionnelle des mandats des sénateurs, des députés provinciaux, gouverneurs et vice-gouverneurs pour l'appliquer à la fonction présidentielle dans l'hypothèse du report des élections présidentielle et législatives de $2016^{71}$.

Qu'adviendrait-il en cas de report des élections en 2016 ? En d'autres termes, que ferait-on lorsque le 20 décembre 2016, si le peuple congolais n'aurait pas élu un nouveau Président de la République ? La solution à cette situation est prévue à l'article 75 de la Constitution. Cette disposition énumère les cas d'ouverture de la vacance à la Présidence de la République. Pour prévenir toutes les manœuvres visant une prolongation du mandat présidentiel, le constituant n'a pas dressé une liste exhaustive de cas menant à la vacance à la fonction du Président de la République. Cette liste est illimitée. Ainsi, l'article 75 dispose :

En cas de vacance pour cause de décès, de démission ou pour toute autre cause d'empêchement définitif, les fonctions de Président de la République, à l'exception de celles mentionnées aux articles 78, 81 et 82 sont provisoirement exercées par le Président du Sénat ${ }^{72}$.

Le caractère non exhaustif de l'énumération de cas d'ouverture de la vacance à la Présidence de la République se manifeste par l'expression « pour toute autre cause d'empêchement définitif ». Dans ce cas précis, la limite des mandats est une des causes d'empêchement définitif pour l'exercice de la fonction présidentielle. Pour répondre à la question cidessus, il convient d'affirmer qu'en cas de report de l'élection présidentielle en 2016, le Président du Sénat assumera les fonctions de Président de la République jusqu'à l'élection

70 Wetsh'okonda Koso M., Les profils des membres de la Cour constitutionnelle de la République démocratique du Congo, http://congosiasa.blogspot.com/2015/05/ (Consulté le 26 juin 2015).

71 Déclaration des Organisations de la Société civile de la République Démocratique du Congo à l'issue du séminaire atelier sur la révision de la Constitution organisé Kinshasa (CEPAS) du 22 au 23 avril 2014.

72 Art. 75 de la Constitution...op. cit. 
du nouveau Président de la République, à intervenir entre 60 et 90 jours au plus. En cas de force majeure, ce délai peut être rallongé à 120 jours, c'est-à-dire à avril 2017.

\section{CONCLUSION}

L'alternance politique démocratique a été consacrée par la Constitution du 18 février 2006 comme une valeur républicaine fondamentale. Elle a été conçue comme un antidote aux crises politiques ayant freiné le développement de la RDC. L'alternance politique est devenue une recette cardinale de la consolidation de la démocratie et de l'Etat de droit tant en RDC que dans d'autres pays africains, plus particulièrement ceux de la région des Grands Lacs. Elle est un gage de la paix et de la sécurité; renvoyant ainsi aux calendes congolaises et africaines tous les fallacieux arguments en faveur du recours à la force armée pour la conquête du pouvoir d'Etat.

Pour ce faire, le constituant de 2006 avait prévu des garanties juridiques pour assurer l'alternance politique en RDC et avait cru avoir réussi à conjurer tous les démons des crises politiques et des conflits armés à l'issue du Dialogue inter congolais. Ces garanties juridiques sont la limitation du nombre et la durée des mandats présidentiels, le statut d'ancien Président de la République et le devoir de résistance à la prise de pouvoir par la force ou à son exercice en violation de la Constitution. Ces garanties ne souffrent d'aucune équivocité.

Malgré la clarté des dispositions constitutionnelles qui portent ces garanties juridiques, celles-ci ont fait l'objet d'atteintes et de tentatives d'atteintes, notamment à l'occasion de la révision constitutionnelle du 20 janvier 2011, à l'élection présidentielle du 28 novembre 2011 et au cours des débats sur les projets de révision constitutionnelle. Devant les intentions attribuées à la majorité au pouvoir de faire sauter le verrou de la limitation des mandats présidentiels, les partis politiques de l'opposition et la Société civile menacent de faire application de l'article 64 de la Constitution.

En tout état de cause, l'alternance politique démocratique est irréversible en RDC. Rien ne peut l'arrêter ni l'énormité des chantiers ni les délices de la politique « révolution de la modernité ». Au cas contraire, le pays risque de basculer dans un cycle des violences qui n'épargnera personne. Ce serait un retournement fâcheux qui anéantirait tous les efforts et souillerait les sacrifices consentis par le peuple congolais depuis un quart de siècle pour remettre le pays sur les rails du processus démocratique. Il appartient au peuple de défendre, par les moyens légitimes à sa disposition, l'alternance politique à travers, l'exigence du respect par tous les dirigeants, de la Constitution du 18 février 2006.

Au-delà des débats politiques, il convient de faire remarquer que la RDC n'est pas allée loin sur sa trajectoire pour son développement politique, économique et social depuis un quart de siècle maintenant. Ces débats sur l'alternance politique durent depuis deux ans. Ils coûtent énormément chers inutilement à l'Etat et au peuple congolais en termes d'énergies, de temps et des moyens. Beaucoup d'énergies sont gaspillées soit pour démontrer qu'il faut qu'il y ait alternance en 2016, soit pour faire barrage à cette alternance, selon le camp dans 
lequel on se trouve. En attendant, les projets de développement stagnent parce que l'attention des gouvernants est focalisée sur leur avenir politique après 2016.

Pour ce faire, les gouvernants ne lésinent pas sur les moyens en vue de mener à terme leurs plans. Il y a également perte en moyens humains dont la RDC a besoin pour son développement. Certains congolais sont placés devant un choix difficile: soit se démobiliser pour mourir en silence sous une domination politique appauvrissante, soit se lever contre le régime impitoyable pour être fauché par les bals des policiers et militaires. Enfin de compte, la RDC n'a pas besoin des manœuvres tendant à empêcher l'alternance démocratique sous-prétexte d'un quelconque dialogue ou des consultations. Ce sont des recettes du passé qui ne résisteront pas devant l'impératif de l'alternance en 2016 si l'on ne veut pas replonger le pays dans la guerre. L'on devait éviter toutes ces manœuvres en respectant la Constitution et ce, pour de la paix et de l'unité nationale.

\section{Références}

Abahlali baseMjondolo, SA xenophobic attacks: A view from below, http://pambazuka.org/en/category /features/94476 (Consulté le 15 avril 2015).

Acte Constitutionnel de transition, J.O.Z, 35 $5^{\mathrm{ème}}$ année, numéro spécial du 9 avril 1994.

AETA, Lettre de la Société civile congolaise au Secrétaire général des Nations Unies sur la crise postélectorale en RDC, Kinshasa, le 07 février 2012.

Akele Adau P., La question de la qualité militaire du Président Joseph Kabila au regard de la loi sur l'identification et l'enrôlement des électeurs, Congo-Afrique (Octobre 2005) n 398, pp. 431-441.

Akele Adau P., Réponses pénales au discours du désordre ou au désordre du discours constitutionnel en République Démocratique du Congo : la Cour constitutionnelle à l'épreuve, Revue africaine de la démocratie et de la gouvernance, Vol. I, n 2 \& 3, 2014, pp. 43-62.

Aziz Salmone Fall, Burkina, vers la libération, http://pambazuka.org/fr/category/features/93299, (Consulté le 03 janvier 2015).

Balingene Kahombo, La Cour suprême de Justice, Cour constitutionnelle transitoire, http://www.la-con stitution-en-afrique.org/, (Consulté le 2 février 2011).

Biaya T.K., Quelle Démocratie pour l'Afrique? Réflexions sur ses Possibilités et ses Caractéristiques, Dakar, 1998.

Boshab Mabuj E., Entre la révision constitutionnelle et l'inanition de la nation, Bruxelles, 2013.

Bruno Jaffré, Burkina Faso : Blaise Compaoré vient de déclarer la guerre à son peuple, http://pambazu ka.org/fr/category/features/93300, (Consulté le 03 janvier 2015).

C.S.J, 16 décembre 2011, RCE 011/PR, inédit.

Conférence Episcopale Nationale du Congo, Le peuple congolais a faim et soif de justice et de paix. Le courage de la vérité (cf. 2 Co 7, 14). Message de l'Assemblée plénière extraordinaire de la CENCO aux fidèles catholiques et à l'ensemble du peuple congolais, Kinshasa, Editions du Secrétariat général de la CENCO, 2012.

Constitution de la République Démocratique du Congo, JORDC, $47^{\text {ème }}$ année, numéro spécial du 18 février 2006. 
CSJ, 13 avril 2006, Arrêt RCDC 003/KIN, en cause MSD contre CEI, in Bulletin des arrêts de la CSJ., pp. 3-5.

CSJ, 15 septembre 2006, Arrêt R.Const. 37/TSR, en cause la CEI, Bulletin des arrêts de la CSJ.

CSJ, 27 novembre 2006, Arrêt RCE.PR. 009, en cause Recours du MLC, Bulletin des arrêts de la CSJ, pp. 83-110.

de Saint Moulain L., Projet de Constitution de la RDC. Dimension sociale, Congo-Afrique, $\mathrm{n}^{\circ} 397$, septembre 2005, pp. 84-94.

Déclaration des Organisations de la Société civile de la République Démocratique du Congo à l'issue du séminaire atelier sur la révision de la Constitution organisé Kinshasa (CEPAS) du 22 au 23 avril 2014.

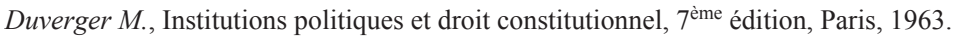

Ellul J., Histoire des institutions politiques, Paris, 1956.

Esambo Kangashe J.L., Le droit constitutionnel, Bruxelles, 2013.

Etekou Bédi Y.S., L'alternance démocratique dans les Etats d'Afrique francophone, Thèse de doctorat, Université de Paris-Est et Université de Cocody, 2013.

Favoreu L. et al., Droit constitutionnel, $9^{\text {ème }}$ édition, Paris, 2006.

http://radiookapi.net/actualite/2015/01/19/rdc-plusieurs-activites-perturbees-kinshasa-dans-les-manifes tations-contre-la-loi-electorale/, (Consulté le 25 avril 2015).

http://unpan1.un.org (Consulté le 25 avril 2015).

http://www.toupie.org/Dictionnnaire/Alternance (Consulté le 19 septembre 2014).

Justice pour Thomas Sankara, Justice pour l'Afrique, Blaise Compaoré chassé par son peuple ! Il ne doit pas échapper à la justice, http://pambazuka.org/fr/category/features/93302, (Consulté le 03 janvier 2015) .

Kabemba C., South Africa in the DRC : renaissance or néo-imperialism?, in Sakhela Buhlungu et al (ed.), State of the Nation. South Africa 2007, Cape Town, 2007, pp. 533-551. Manby B., La nationalité en Afrique, Paris, 2011.

Kamukuny Mukinay A., Contribution à l'étude de la fraude en droit constitutionnel congolais, Thèse de doctorat, Faculté de droit, Université de Kinshasa, 2007.

Kaniki F., Discours de Barack OBAMA : Une arme pour l'alternance politique en Afrique,. http://afriq ue.kongotimes.info/afrique (Consulté le 29 avril 2015).

Kibangula T., Burundi : au 3ème jour de manifestations, le pouvoir de Nkurunziza parle d'"insurrection", http://www.jeuneafrique.com/Article/, (Consulté le 30 avril 2015).

Kifwabala Tekilazaya, Fataki wa Luhindi D. et Wetsh'okonda Koso M., République Démocratique du Congo. Le secteur de la justice et l'Etat de droit, Johannesbourg, 2013.

Le Potentiel, Résultats des élections 2011. Monsengwo jette de l'huile sur le feu, Le Potentiel, $\mathrm{n}^{\circ} 5402$ du mardi 13 décembre 2011.

Loi fondamentale du 19 mai 1960 relative aux structures du Congo, Moniteur congolais, $\mathrm{n}^{\circ} 1$ de mai 1960.

Lumingu J.C., Evariste Boshab livre la raison de la révision constitutionnelle, L'Etendard des Révélateurs, $n^{\circ} 00$ du 31 janvier au 6 février 2011. 
Mampuya Kanunk'a-Tshiabo A., Le respect des textes légaux comme problème d'éthique de gouvernance au Congo, Revue de l'Université du Kasayi, Vol. 1, n 1, Février 2013, pp. 145-165.

Mbata Betukumesu Mangu A., Mandats présidentiels et révisions constitutionnelles en Afrique. La République Démocratique du Congo dans la perspective 2016, Kinshasa, 2013.

Mboya Mzobanzi M., Mbeki and the Peace Process in Africa : A Contribution to Africa's Renaissance, International Journal of African Renaissance Studies, Volume 1, N 1, 2006, pp. 80-89.

Mission d'observation électorale de l'Union européenne en République Démocratique du Congo, Rapport final des élections présidentielle et législatives du 28 novembre 2011, www.moeue-rdc.eu (Consulté le 21 novembre 2011).

Mission nationale d'observation, Rapport à mi-parcours de l'observation des élections du 28 novembre 2011, Kinshasa, le 17 décembre 2011.

Mobutu, Dignité pour l'Afrique. Entretiens avec Jean-Louis Remilleux, Paris, 1989.

Mudimbe V.Y., Shaba deux. Les Carnets de Mère Marie Gertrude, Paris, 1989.

Mumbere Bamlango D., Des actes législatifs et du pouvoir régulateur : quand la Cour suprême de Justice donne une portée expansive aux attributions de la Cour constitutionnelle, Horizons, Revue de droit et science politique du Graben, $\mathrm{n}^{\circ}$ 5, pp. 46-58.

Ngapi R., En soutien à la déclaration du Cardinal Monsengwo. Le clergé catholique kinois : 'La vérité vous rendra libres', Le Potentiel, n 5407 du Lundi 19 décembre 2011.

Ntumba Luaba, Le projet de la Constitution de la République du Zaïre, Zaïre- Afrique, 1993, n³10.

Projet de Constitution de la République fédérale du Congo, Palais du Peuple, Kinshasa, 1992.

Quermone J.L., L'alternance au pouvoir, Paris, 2003.

Radio Okapi, Révision de la constitution : faut-il toucher les articles irréformables?, http://radiookapi.n et/emissions-2/ (Consulté le 30 avril 2015).

Sall E., CODESRIA Acceptance Speech, CODESRIA Bulletin, Numbers 3 \& 4, 2013.

Sesanga Hipungu D., La voie du changement. Un pari de la raison pour la Rd Congo, Paris, 2011.

Vunduawe te Pemako F. et Mboko Dj'andima J.M., Droit constitutionnel du Congo. Textes et documents fondamentaux, Vol. 2, Bruxelles, 2013.

Vunduawe te Pemako F. et Mboko Dj'Andima J.M., Droit constitutionnel du Congo. Textes et documents fondamentaux, Vol. 2, Bruxelles, 2013.

Wetsh'okonda Koso M., Les profils des membres de la Cour constitutionnelle de la République démocratique du Congo, http://congosiasa.blogspot.com/2015/05/ (Consulté le 26 juin 2015).

Wetsh'okonda Koso M., Les textes constitutionnels congolais annotés, Kinshasa, 2010. 